\title{
Lumen
}

Selected Proceedings from the Canadian Society for Eighteenth-Century Studies

\section{Marques d'ironie dans les Avantures de Monsieur Robert Chevalier, dit de Beauchêne d'Alain-René Lesage}

\section{Emmanuel Bouchard}

Volume 26, 2007

Imitation et invention au siècle des Lumières

Imitation and Invention in the Eighteenth Century

URI : https://id.erudit.org/iderudit/1012058ar

DOI : https://doi.org/10.7202/1012058ar

Aller au sommaire du numéro

Éditeur(s)

Canadian Society for Eighteenth-Century Studies / Société canadienne d'étude du dix-huitième siècle

ISSN

1209-3696 (imprimé)

1927-8284 (numérique)

Découvrir la revue

Citer cet article

Bouchard, E. (2007). Marques d'ironie dans les Avantures de Monsieur Robert Chevalier, dit de Beauchêne d'Alain-René Lesage. Lumen, 26, 21-33.

https://doi.org/10.7202/1012058ar

Copyright (c) Canadian Society for Eighteenth-Century Studies / Sociéte canadienne d'étude du dix-huitième siècle, 2007
Ce document est protégé par la loi sur le droit d'auteur. L'utilisation des services d'Érudit (y compris la reproduction) est assujettie à sa politique d'utilisation que vous pouvez consulter en ligne.

https://apropos.erudit.org/fr/usagers/politique-dutilisation/ 


\section{Marques d'ironie dans les Avantures de Monsieur Robert Chevalier, dit de Beauchêne d'Alain-René Lesage}

L'ironie fait partie de ces traits d'écriture qui, dans la production romanesque de Lesage, ne se sont jamais démentis ; il faudrait même dire qu'elle constitue le moteur de son œuvre et affirmer, avec Roger Laufer, que «Lesage est grand écrivain par ironie ${ }^{1} »$. Dans les Avantures de Monsieur Robert Chevalier, dit de Beauchêne ${ }^{2}$, Lesage est conscient des poncifs $\mathrm{qu}^{\prime} \mathrm{il}$ assemble, forgeant, pour reprendre une fois de plus l'expression de Roger Laufer, «une littérature de littérature ${ }^{3}$ »; la distance ironique l'illustre bien, en même temps qu'elle constitue l'originalité de l'œuvre.

Lesage traduit, emprunte, remanie, adapte; presque toutes ses œuvres en témoignent, des Lettres galantes d'Aristénète aux romans de source espagnole, en passant par les contes orientaux des Mille et un jours. Dans ses meilleures pages, il renouvelle les sources et les lieux communs dont il se nourrit; cette liberté, qui est une dimension essentielle de l'invention chez Lesage et qui se déploie de façon particulièrement convaincante dans les Avantures de Monsieur Robert Chevalier, constituera l'objet de notre commentaire. Mais avant d'entrer dans l'œuvre, qu'il nous soit permis de fixer les bases sur lesquelles reposera notre lecture.

1 Roger Laufer, Lesage ou le métier de romancier, Paris, Gallimard, coll. «Bibliothèques des idées", 1971, p. 385.

2 Lesage, Les Avantures de Monsieur Robert Chevalier, dit de Beauchêne, capitaine de flibustiers dans la nouvelle France, Paris, Ganeau, 1732. Dans la suite, nous désignerons cette œuvre par l'abréviation Avantures.

3 R. Laufer, Ibid., p. 8. 
L'ironie procède par antithèse. Les définitions les plus courantes insistent assez sur l'opposition entre énoncé et intention qui la caractérise : «opposition transparente entre ce qui est littéralement et ce qui est vraiment dit $^{4} »$, écrit Beda Alleman. Celle à laquelle nous nous intéressons ne saurait toutefois s'y limiter et il faut la concevoir davantage comme une distanciation entre énoncé et intention: le discours ironique fait entendre autre chose que ce que disent les mots ${ }^{5}$. Devant l'énoncé ou, plus largement, la situation ironique - qu'on pourrait définir dans un roman comme un ensemble d'événements ou d'actions -, l'accès à cette autre chose nécessite une connaissance minimale de ce que César Dumarsais, un contemporain de Lesage, appelle, à la suite de Port-Royal, les «idées accessoires ${ }^{6}$ » (le contexte d'énonciation, qui comprend la situation de l'auteur, mais surtout le genre, l'esthétique du texte, voire d'autres textes). Assurément, Lesage pratique l'ironie à partir de ses propres énoncés, jouant allègrement de l'antithèse dans de nombreuses saillies que nous n'étudierons pas ici. Il est cependant plus grand ironiste lorsqu'il sollicite les énoncés des autres, et cela à différents degrés : les autres textes ou les autres auteurs, lorsqu'il s'en moque directement ; la tradition romanesque, la tradition de la relation de voyage et ses poncifs, lorsqu'il s'en écarte ou en inverse les contenus ; enfin, la tradition historique, lorsqu'il la réécrit.

Notre analyse se fonde essentiellement sur cette conception de l'ironie comme décalage ou, pour reprendre les mots de Beda Alleman, comme "champs de tensions ${ }^{7}$ ". Ces tensions ironiques prennent diffé-

4 Beda Alleman, «De l'ironie en tant que principe littéraire», Poétique, 36, novembre 1978, p. 389.

5 Voir la définition de Clausier citée par Florence Mercier-Leca, L'ironie, Paris, Hachette, coll. «Ancrages", 2003, p. 13.

6 Cité dans Philippe Hamon, L'ironie littéraire. Essai sur les formes de l'écriture oblique, Paris, Hachette, coll. «Recherches littéraires», 1996, p. 21. Voir encore Beda Alleman, qui explique plus en détail les difficultés de perception de l'ironie: «Constamment, ce qu'il y a d'ironique dans un texte ne ressort qu'à partir du contexte, au sens le plus large de ce terme encore peu éclairci, et, bien entendu, sans qu'il y ait renvoi à ce contexte par le moyen de signaux (perceptibles). L'arrière-plan ironique de ce qui est dit littéralement est toujours déjà donné sous forme de présupposé (vorausgesetzt) : sinon, ce qui est dit littéralement serait compris comme littéral et non pas comme ironique. En même temps, les signaux formels sont interdits, c'est-à-dire que l'arrière-plan devra être présupposé de façon aussi tacite que possible : car toute mesure prise de façon que le lecteur aille donner du nez contre l'arrière-plan annule l'effet ironique» («De l'ironie en tant que principe littéraire», art. cit., p. 390391).

7 Beda Alleman, «De l'ironie en tant que principe littéraire», art. cit., p. 396. 
rentes formes, comme le résume bien Philippe Hamon dans son essai sur l'ironie littéraire :

1. tension entre deux parties disjointes et explicites du même énoncé (deux registres, deux champs sémantiques, deux termes d'une comparaison, mis en voisinage hétéroclite) ;

2. tension entre le narrateur et son propre énoncé, dont il se désolidarise entièrement ou partiellement ;

3. tension entre l'énoncé et un autre énoncé extérieur, cité, parodié, pastiché ou simplement mentionné en échos («mimèse») ;

4. tension entre le discontinu et le continu, par introduction de degrés là où il n'y en a pas, ou par neutralisation de degrés préexistants ${ }^{8}$.

Les quelques scènes que nous étudierons entrent dans l'une ou l'autre des trois dernières catégories de tensions présentées par Philippe Hamon: elles engagent le narrateur dans la relation qu'il entretient avec lui-même ou, pourrions-nous dire, avec le héros; elles appellent d'autres textes et, par là, les traditions littéraire et historique. Nous examinerons ici deux lieux communs de la relation de voyage à laquelle l'œuvre de Lesage est largement redevable: la cruauté iroquoise et l'anthropophagie.

\section{La cruauté iroquoise}

Dans son récit, l'un des deux narrateurs du roman, Robert Chevalier, récupère par endroits les lieux communs de l'ethnographie pour mieux les remanier, allant dans les meilleurs cas jusqu'à en inverser complètement la logique par ironie ou par autodérision. Dans le récit qu'il fait de sa jeunesse en Nouvelle-France, il ne manque pas d'abord de se ranger à ce que disent du peuple iroquois la plupart des voyageurs et romanciers depuis plusieurs décennies :

Ces Sauvages gagnez par les présens des Anglois, faisoient quelquefois des courses jusqu'aux portes de Montreal. Ils entroient dans le pays par pelotons, se tenoient cachez dans les bois pendant le jour, se rassembloient la nuit, \& venoient fondre sur quelque Village. Ils le pilloient, puis se retiroient promptement avec leur butin, après avoir mis le feu aux choses qu'ils ne pouvoient emporter. Mais ils avoient grand soin surtout de ne pas oublier les chevelures

8 Philippe Hamon, L'ironie littéraire, op. cit., p. 40. 
de ceux qu'ils avoient tuez. Je les ai souvent vû couper de ces chevelures, \& sans contredit ils s'y prennent plus adroitement que les Barbiers d'Europe pour ne point perdre de cheveux, puisqu'ils arrachent en même-tems la peau de dessus le crane. [...] Voilà les drapeaux qu'ils aiment à prendre sur leurs ennemis (Avantures, t. I, p. 6-8).

Alliance avec les Anglais, expéditions surprises en Nouvelle-France, pillage et scalp font partie des principales composantes de la geste iroquoise ; elles suffisent à laisser voir au lecteur un portrait plutôt sombre de ces Amérindiens que la Nouvelle-France redoute depuis longtemps. «Les Iroquois [...] sont des peuples sauvages très-belliqueux», écrit Lahontan en 1703 dans ses Nouveaux voyages ${ }^{9}$; on pourrait continuer et tirer des remarques à peu près semblables des textes de Bacqueville de la Potherie, de Claude Lebeau, du jésuite Charlevoix et de bien d'autres relateurs ou romanciers de l'époque.

Sans interrompre sa narration, c'est-à-dire en se servant d'événements plus que de descriptions, Lesage installe le lieu commun dans son récit pour mieux s'en distancier par la suite. Il invoque notamment la figure du chef iroquois la Chaudière Noire, un «terrible mortel» qui, à la fin du XVII ${ }^{\mathrm{e}}$ siècle, est «la terreur du Canada» (Avantures, t. I, p. 8). L'introduction de cette figure dans le récit concrétise par l'exemple la violence et la cruauté des Iroquois ${ }^{10}$; elle permet encore au narrateur de se mettre en scène et a fortiori de valoriser sa position, son action. Car,

9 Lahontan, CEuvres complètes, éd. établie par Réal Ouellet et Alain Beaulieu, Montréal, Presses de l'Université de Montréal, coll. «Bibliothèque du Nouveau Monde», 1990, t. I, p. 257.

10 Ce caractère iroquois demeure bien souvent assez général dans certains textes historiographiques ou relations de voyage. $\mathrm{Qu}^{\prime}$ on lise, pour s'en convaincre, les premières pages du chapitre sur la guerre des Mours des sauvages américains de Joseph-François Lafitau, qui révèlent une tendance à l'analyse et à la conjoncture plus propres à l'ethnologue qu'au romancier: "Les Hommes, qui sont si desœuvrés dans leurs Villages, ne se font une gloire de leur indolence que pour donner à entendre qu'ils ne sont proprement nez que pour les grandes choses, \& sur-tout pour la Guerre, laquelle exposant leur courage aux plus rudes épreuves, leur fournit de frequentes occasions de mettre dans son plus beau jour toute la noblesse de leurs sentimens, \& l'inébranlable fermeté d'une grandeur d'ame vraïement heroïque. La chasse \& la pêche, qui après la guerre emporte toute leur attention, ne leur sont agreables, que parce qu'elles en sont l'image, \& peut-être en laisseroient-ils le soin aux femmes, ainsi que de la nourriture \& de tout le reste, si elles n'étoient en même-temps un exercice qui les forme à se rendre terribles à des ennemis encore plus redoutables, que ne le sont les bêtes feroces» (Mours des sauvages amériquains, comparées aux mours des premiers temps, Paris, Saugrain l'aîné et Charles Estienne Hochereau, 1724, t. II, p. 161). 
évidemment, Robert Chevalier n'entend pas demeurer inactif devant la violence des Iroquois et de leur chef. Le tempérament du héros canadien le pousse naturellement vers le combat. Jeune, mais déjà emporté et violent, «toujours prêt à frapper»(Avantures, t. I, p. 3), Robert Chevalier trouve l'occasion d'être fait prisonnier par les Iroquois alors que ceux-ci font une incursion à Montréal en 1689. C'est l'épisode historique que Lahontan, Bacqueville de la Potherie et les historiographes après eux ont appelé le «massacre» de Lachine, rapporté dans la suite du texte :

Les Sauvages firent leur coup en moins d'un quart d'heure. Ils tuérent une trentainedepersonnes, avantqu' on fûtenétatdeles repousser, mirentlefeuà plusieurs maisons, \& se retirerent avec un butin plus gros que riche, \& quelques prisonniers, parmi lesquels mon frere aîné eut le malheur de se trouver. Comme je cherchois des yeux les Iroquois, j'en apperçus douze ou quinze qui démeubloient une maison avant que de la bruler, \& qui en enlevoient deux petits enfans. Je criai aussitôt à pleine tête : Quartier, Messieurs, quartier! Je me rends ; emmenez-moi avec vous.

Je ne sçai s'ils m'entendirent mais je me présentai à eux de si bonne grace, qu'ils ne purent me refuser la satisfaction d'être leur prisonnier. L'un d'entre eux me prit sur ses épaules, \& nous rejoignîmes promptement le gros de la troupe (Avantures, t. I, p. 11-13).

À un moment où les habitants de la colonie et les autorités elles-mêmes craignent les Iroquois plus que quiconque, au milieu de «la consternation [...] générale ${ }^{11}$ » provoquée par les «cruautez inouies ${ }^{12}{ }$ des "Barbares ${ }^{13}$ ", le futur flibustier offre volontairement sa liberté aux assaillants. Comme personnage de roman, il s'oppose au comportement général, à la méfiance et à la peur de la population qu'il observe autour de lui ; comme narrateur, Robert Chevalier - et, à travers lui, le romancier - s'écarte des poncifs, auxquels il substitue une situation inhabituelle dans la relation de voyage, une mise en scène résolument romanesque, au sens où elle tient peu compte de la vérité documentaire et qu'elle glorifie d'autant son protagoniste. Parce que l'invraisemblance traverse cette scène de reddition volontaire du jeune Chevalier, il faut la lire comme une réponse à d'autres scènes ou, du moins, à certains lieux communs qu'ont largement contribué à diffuser les relations de

11 Lahontan, QEuvres completes, op. cit., t. I, p. 442.

12 Bacqueville de la Potherie, Histoire de l'Amérique septentrionale. Divisée en quatre Tomes, Paris, Jean-Luc Nion et François Didot, 1722, t. III, p. 59.

13 Lahontan, OEuvres completes, op. cit., t. I, p. 443. 
voyage depuis le XVII siècle. Cette réponse utilise les moyens du roman, qu' on trouve ailleurs dans le texte, et même dans d'autres œuvres de Lesage : le remaniement ironique, cette mise en tension de l'anecdote représentée et de l'événement historique, qui, après la singularisation du trait ethnographique par l'exemple, constitue le deuxième mode de distanciation.

\section{L'anthropophagie}

La violence des Iroquois trouve, chez Lesage, une résonance dans un autre lieu commun de la relation de voyage ou du texte utopique : celui de l'anthropophagie, qui permet d'illustrer une fois de plus la dimension ironique de la distanciation. Dans le premier livre du roman, après ses premières sorties en mer sur le vaisseau de Pierre Morpain, Robert Chevalier débarque sur l'île de Saint-Domingue, où il est accueilli par un Français, M. de Rémoussin, qui le présente à sa femme et à quelquesunes de ses parentes:

Voici un Sauvage curieux que je vous amene. Sans aller au Canada, vous allez voir un Iroquois, mais un Iroquois qui ne vous fera pas peur. À ce mot d'Iroquois, les Dames se formant une idée de monstre, fait à peu près comme leurs Négres, s'avancerent pour me considérer, \& ce ne fut pas sans étonnement qu'elles virent un gros garçon d'assez bonne mine, blanc \& blond comme le sont communément les Canadiens (Avantures, t. I, p. 100-101).

Lesage brouille les questions identitaires, en multipliant les lieux communs et en les opposant les uns aux autres (l'aspect physique des Canadiens et des Iroquois). Mais il y a plus encore dans le portrait du «Sauvage curieux». Après avoir découvert la naissance et l'histoire de Robert Chevalier, Mme de Rémoussin continue de l'interroger :

Madame de Rémoussin étonnée de me voir dans un âge si peu avancé ne respirer que les combats, $m$ 'en fit des reproches, en me demandant malicieusement combien j'avois mangé d'Anglois depuis que je courois les Mers, ne doutant point que je ne fusse assez inhumain pour suivre la coutume des Sauvages, qui disent qu'un ennemi vaincu augmente personnellement leurs provisions de bouche (Avantures, t. I, p. 103).

La question de l'anthropophagie préoccupe, autant que Mme Rémoussin, les observateurs et les voyageurs depuis plusieurs années, voire plusieurs siècles. Elle constitue presque toujours la meilleure preuve de 
la cruauté que l'on attribue aux peuples américains ${ }^{14}$, antillais ${ }^{15}$ ou africains $^{16}$. Les scènes d'anthropophagie abondent dans les relations des voyageurs et dans les textes historiographiques ${ }^{17}$. Lorsqu'il s'agit des Iroquois, ou généralement des Amérindiens, Robert Chevalier ne fait pour sa part mention de l'anthropophagie qu'indirectement, à travers le discours de Mme de Rémoussin. Cette réserve est-elle due au sentiment qu'il a d'appartenir lui-même à cette nation cruelle? Est-ce pour lui une façon de se défendre ou de conserver sa dignité que de présenter l'anthropophagie des Amérindiens comme le préjugé d'un Français immigré aux Iles de l'Amérique ? Peut-être, mais, dans l'ensemble de l'œuvre, la question dépasse les intérêts du flibustier et il semble bien qu'elle serve également le discours d'un autre personnage, celui de

14 Les exemples sont nombreux. On ne citera que le jésuite Paul Lejeune, qui écrit, dans sa Relation de 1632, à propos des prisonniers faits par les Amérindiens qu'il rencontre à Tadoussac : "Quand ils les font mourir ils les attachent à un poteau, puis les filles aussi bien que les hommes leur appliquent des tisons ardents et flambans aux parties les plus sensibles du corps, aux costez, aux cuisses, à la poitrine, $\&$ en plusieurs autres endroits : ils leurs levent la peau de la teste, puis jettent sur le crane ou le test découvert, du sablon tout bruslant; ils leurs percent les bras au poignet avec des bastons pointus, \& leurs arrachent les nerfs par ces trous. Bref ils les font souffrir tout ce que la cruauté et le Diable leur met en l'esprit. En fin pour derniere catastrophe ils les mangent et les devorent quasi tout crus» (Reuben Gold Thwaites (dir.), The Jesuit Relations and Allied Documents. Travels and Explorations of the Jesuit Missionaries in New France, 1610-1791, New York, Pageant Book, 1959, t. 5, p. 29-30; voir également t. 34, p. 146 ; et Chrestien Leclercq, Nouvelle relation de la Gaspésie, éd. critique par Réal Ouellet, Montréal, Presses de l’Université de Montréal, coll. «Bibliothèque du Nouveau Monde», 1999, p. 521-522).

15 Voir Jean-Baptiste Du Tertre, Histoire générale des îles de Saint-Christophe, Paris, Jacques Langlois, 1654, p. 449-452 ; Jacques Bouton, Relation de l'establissement des François depuis l'an 1635 En l'isle de la Martinique, l'une des Antilles de l'Amerique. Des meurs des Sauzages, de la situation, \& des autres singularitez de l'isle, Paris, Sébastien Cramoisy, 1640, p. 35 ; Adrien Le Breton, Description de l'Isle de Saint-Vincent, texte établi et présenté par Robert Pinchon, Annales des Antilles, 9, 1961, p. 44.

16 Voir Frank Lestringant, Le Cannibale : grandeur et décadence, Paris, Perrin, coll. «Histoire et décadence", 1994, $319 \mathrm{p}$.

17 Bacqueville de la Potherie, par exemple, réserve à ce trait ethnographique la conclusion d'une lettre de son Histoire de l'Amérique septentrionale, dans laquelle il s'emploie à décrire le caractère de la nation iroquoise. Au terme d'un tableau des supplices appliqués aux prisonniers ennemis, il note: "L'on ne meurt pas d'abord de tous [sic] ces sortes de tourmens que l'on exerce à plaisir sur eux : enfin aussi-tôt que cette victime a expiré, ils lui arrachent le cœur ; ils suçent le sang, \& coupent le corps en plusieurs morceaux qu'ils mangent. Tel est le caractere de la plus redoutable nation qui soit dans l'Amerique, qui d'ailleurs sont trés humains \& trés genereux avec ceux qui deviennent leurs amis» (op. cit., t. III, p. 49-50). 
Mlle Duclos, que rencontre le comte de Monneville lors de son voyage en Nouvelle-France. Arrivé dans le village huron dont Mlle Duclos est devenue sakgame, Monneville a tout lieu d'observer l'empire qu'a pris sur le village et ses habitants cette Française, qui n'hésite pas à défendre certaines coutumes autochtones :

Les Hurons, a-t-on dit, ont tué, ont mangé les prisonniers qu'ils ont faits quand on a tenté des descentes sur leurs côtes. Ce sont donc les Sauvages les plus cruels, des Anthropophages, des Monstres [...] Eh ! bon Dieu devoient-ils faire autrement ? Jugeons-en sans prévention.

Il voyent arriver chez eux des ennemis qui n'ont à leurs yeux rien que de terrible, de monstrueux, de surnaturel, [...] pourquoi ne les tueront-ils pas pour s'en défaire ? [...] Oüi, mais, dira-t-on, pourquoi [les] manger ? Hé, pour quelle raison voulez-vous qu'ils ne [les] mangent pas ? C'est leur coutume de traiter ainsi les ennemis qu'ils peuvent prendre. Trouverions-nous bien raisonnable un Chasseur qui n'ayant jamais vû que des perdrix rouges n'en tueroit pas une grise qui viendroit dans son Canton, ou qui l'ayant tuée \& la voyant grosse \& grasse l'enfoüiroit plutôt que de la manger ? Nous ne jugerions jamais témerairement si laissant là nos préjugez, nous nous mettions à la place de ceux de qui nous voulons être les Juges (Avantures, t. II, p. 83-85).

Ici, l'écriture lesagienne utilise l'anthropophagie des Amérindiens au profit d'un discours plus vaste - et plus fondamental - mettant de l'avant le relativisme culturel : les Hurons mangent leurs prisonniers par respect pour des coutumes ancestrales, comme un Européen qui perpétuerait tel ou tel usage. C'est l'attachement à la tradition que soutient Mlle Duclos plus que le contenu de la tradition proprement dite. Ce qui paraît étrange pour l'Européen ne l'est pas pour l'Américain et vice versa. Dans la suite du texte, Mlle Duclos reprend le motif, bien connu depuis Lahontan et Montesquieu ${ }^{18}$, du visiteur visité : elle imagine les questionnements et l'étonnement d'un Amérindien qui débarque en Europe.

Défendre le relativisme des habitudes culturelles par le moyen de l'anthropophagie, coutume bien plus difficilement admissible que beaucoup d'autres à l'époque, peut apparaître téméraire. Comme le faisait Jonathan Swift trois ans auparavant avec sa Modest Proposal dans laquelle il suggérait au peuple irlandais de vendre ses enfants comme nourriture, interprétant au pied de la lettre la métaphore des

18 Et repris dans les Lettres iroquoises de Maubert de Gouvest (1752), pour ne citer qu'un exemple. La forme question-réponse rappelle celle des Dialogues de Lahon$\tan$. 
riches qui se nourrissent des pauvres, Lesage ébranle la doxa en plaidant d'une manière plus ou moins sérieuse la légitimité d'une pratique qui hante l'imaginaire des voyageurs et des lecteurs ${ }^{19}$; comme ailleurs dans l'œuvre, les moyens (le ton et l'exemple choisi) semblent plus légers que l'intention; la tension est palpable.

Les circonstances dans lesquelles apparait encore le thème de l'anthropophagie sauvage, à propos cette fois des Africains, révèlent de semblables effets ironiques. L'épisode africain survient à la suite d'une tentative d'évasion manquée de Robert Chevalier et de quelques flibustiers prisonniers - dont le comte de Monneville - sur un vaisseau anglais. Pendant la nuit, le narrateur et ses amis élaborent une stratégie pour prendre en otage les membres de l'équipage, mais l'alarme est donnée et les mutins doivent céder la victoire aux Anglais qui, en raison de l'intervention de M. Cazali, un Français sympathique à leur cause, acceptent d'épargner la pendaison à Chevalier et à ses hommes ; les Anglais les abandonneront plutôt sur les côtes de la Guinée où, selon le mot de Cazali, «s'[ils échappent] aux griffes des lions, ce sera pour mourir de faim, ou pour apaiser celle des Negres» (Avantures, t. II, p. 265-266).

Cet avertissement n'est pas sans effrayer les flibustiers qui, laissés à eux-mêmes au milieu d'un désert inconnu, sans autre ressource que leur expérience de marins, manifestent à quelques reprises par la suite la crainte d'être «dévorés par les Negres ou par les bêtes feroces» (Avantures, t. II, p. 266) ; il s'agit en fait de leur principale préoccupation $^{20}$. Cependant, leur première rencontre avec les «Nègres» apaisera leur inquiétude ; ceux-ci sont «poltrons» (Avantures, t. II, p. 277) et "peu aguerris» (Avantures, t. II, p. 281) :

19 Dans le chapitre « Des cannibales » des Essais, Montaigne évoque lui aussi l'anthropophagie des nations "barbares " pour remettre en cause certains usages européens: "Je ne suis pas marry que nous remerquons l'horreur barbaresque qu'il y a en une telle action, mais ouy bien dequoy, jugeans bien de leurs fautes, nous soyons si aveuglez aux nostres. Je pense qu'il y a plus de barbarie à manger un homme vivant qu'à le manger mort, à deschirer par tourmens et par geénes un corps encore plein de sentiment, le faire rostir par le menu, le faire mordre et meurtrir aux chiens et aux pourceaux (comme nous l'avons non seulement leu, mais veu de fresche memoire, non entre des ennemis anciens, mais entre des voisins et concitoyens, et, qui pis est, sous pretexte de pieté et de religion), que de le rostir et manger après qu'il est trespassé " (Montaigne, "Des cannibales ", Essais. Livre I, éd. préparée Alexandre Micha, Paris, Garnier, coll. "Garnier-Flammarion », 1969, p. 258). 
On peut juger par un petit incident que je vais rapporter, combien ces peuples sont peu aguerris. Ma massuë me glissa des mains par hazard, je me baissai avec vivacité pour la ramasser; \& ce mouvement que je fis leur causa tant d'épouvante, qu'ils s'enfuirent presque tous. Vous eussiez vû ceux qui étoient sur les arbres se jetter promptement en bas pour se sauver, de même que si une armée d'Ennemis fût venue fondre sur eux (Avantures, t. II, p. 281-282).

Cette anecdote, comme le reste de la rencontre, montre des autochtones qui représentent l'exact contraire de la cruauté imaginée par M. de Cazali ou par Robert Chevalier et ses hommes. On peut même lire la scène du repas offert aux flibustiers affamés, qui suit ce passage, comme une négation du lieu commun sur l'anthropophagie: non seulement les «Nègres» ne mangent pas les Blancs, mais ils leur offrent généreusement de quoi manger ; non de la chair humaine, mais du poisson.

Mais là où les Africains se distancient davantage de l'image cruelle et anthropophage que leur associaient les flibustiers, c'est lorsqu'ils prennent eux-mêmes la peine de les mettre en garde contre le danger de leurs propres ennemis :

Comme je leur nommai plusieurs fois le Cap-Corse \& Juda pour leur en demander le chemin \& la distance, ils me répondirent par leurs gestes que la route de Juda n'étoit pas pratiquable par terre, \& qu'il nous falloit seulement cinq tours de soleil pour nous rendre au Cap-Corse ; mais qu'à la fin du premier jour nous trouverions un village de Negres avec lesquels ils étoient en guerre, qui étoient les plus méchans du Pays, \& qui nous mangeroient infailliblement (Avantures, t. II, p. 282-283).

Le procédé brille par sa finesse: un Africain entretient la méfiance d'un Blanc à l'endroit d'un autre Africain, plus dangereux que lui parce qu'anthropophage. L'écriture de Lesage joue sur deux plans à la fois : d'une part, elle ridiculise une croyance populaire ou l'obsession de cette croyance qui paralyse littéralement les flibustiers ${ }^{21}$; d'autre part, elle renforce l'authenticité ou, du moins, la vérité romanesque de

21 Le valeureux Robert Chevalier ira jusqu'à verser des larmes tellement sa peur est grande : «Je les priai après cela de se reposer sans crainte, tandis que je veillerois le premier, ce qu'ils refuserent de faire. Je me couchai donc pour leur donner l'exemple, \& je leur dis de m'éveiller lors qu'ils voudroient dormir à leur tour. Je ne me sentois pas plus disposé qu'eux à prendre du repos; mais je ne voulois pas qu'ils s'aperçussent qu'en tâchant de les rassurer, je n'étois pas moins effrayé qu'eux. Leurs plaintes m'attendrissoient \& j'avois le visage couvert de larmes que je cachois en croisant mes mains sur mon front. C'étoit pour la seconde fois de ma vie qu'il m'arrivoit de pleurer» (Avantures, t. II, p. 270-271). 
cette croyance en faisant du «Nègre» à la fois l'objet et le témoin de l'anthropophagie. On trouve encore dans cette scène la présence du quatrième type de tension ironique dont parle Philippe Hamon : dans l'échelle de la cruauté, Lesage introduit des «degrés» entre les Africains (les anthropophages et les non-anthropophages), en même temps qu'il abolit ceux existant entre les Blancs, civilisés, et les Africains, dont plusieurs craignent eux aussi l'anthropophagie.

Par son discours, le Noir confirme en partie ce qu'imaginaient les Blancs. Par ses actions, il n'en est pourtant rien, puisque nulle part dans cet épisode africain l'anthropophagie ou même la cruauté des «Nègres» - sa première phase - n'est représentée en tant qu'action réellement accomplie; elle n'appartient qu'à l'univers du discours, plus précisément à celui du discours préfabriqué, du préjugé. Ce qui est autrement remarquable, c'est que l'endroit et les circonstances où l'anthropophagie s'approche de sa réalisation effective ne sont absolument pas ceux que l'on attendait. Quittant le village, les flibustiers marchent pour se rendre au Cap Corse, accablés par la chaleur, mais plus encore par le fardeau que représentent deux de leurs compagnons malades, qui ont du mal à se traîner et qui ralentissent le groupe. Juste avant de mourir, l'un deux fait connaître aux autres ses derniers souhaits :

je vais mourir content si vous me promettez d'executer ce que je vais vous dire. Au nom de Dieu, que ma mort vous devienne utile. Ne périssez pas de faim de propos deliberé dans ces deserts pour deux ou trois jours de chemin qu'il vous reste à faire. N'épargnez point ma chair, vous en pourrez manger dans un moment \& emporter le reste (Avantures, t. II, p. 292).

Le refus de Robert Chevalier de satisfaire les volontés du mourant comme sa colère contre Monneville, que le désespoir pousse à de semblables propositions, importent moins que l'essence même de cette scène pathétique : la tentation du cannibalisme chez les flibustiers.

Dans un roman où l'anthropophagie sauvage prend une place si marquée dans l'esprit de certains personnages, au point où ses représentations mentales, en plus de se multiplier, agissent souvent comme embrayeurs romanesques - par la crainte qu'elles suscitent, elles dirigent littéralement les actions des flibustiers -, l'inscription dans le réel immédiat de cet acte barbare et répréhensible n'implique ni des Amérindiens, ni des «Nègres», mais les flibustiers eux-mêmes. Les Amérindiens et les Africains de Lesage ne sont cannibales que dans l'opinion commune, facilement disqualifiée par le discours lui-même ou ironiquement remise en cause par les prétentions et les motivations 
de certains personnages qui n'appartiennent pas (tout à fait) au monde sauvage, les flibustiers.

Il est sûr que la violence et la cruauté dont Lesage pare son héros, Robert Chevalier, contribuent à le confondre avec les peuples sauvages mis en scène dans le roman ou, du moins, à le rapprocher de ceux-ci. De là à faire des flibustiers des anthropophages, le pas est grand et Lesage ne le franchit pas; reste pourtant que la tentation est présente dans cet épisode et, comme il le fait souvent, Lesage se dirige vers une voie sans $s^{\prime} y$ engager complètement. «Toute ironie est la construction sémiotique d'une posture d'énonciation visant à un effet», écrit Philippe Hamon ${ }^{22}$, et c'est bien cette posture dans laquelle Lesage se place souvent comme romancier, préférant le sourire à l'attaque directe.

L'œuvre de Lesage fait de l'aventure sa première préoccupation, en entretenant le souci évident de colorer ethnographiquement et historiquement l'«évenement extraordinaire et surprenant» dont parle l'Encyclopédie. L'inversion d'images qui, en 1732, prennent de plus en plus de place dans l'imaginaire collectif procède de l'ironie, tout autant que la disqualification de figures historiques (Montauban, Jacques Cassard, etc.) que des œuvres comme celles d'Exquemelin avaient rendues célèbres et dont nous n'avons pas parlé ici. Comme il le fait ailleurs, dans Le Diable boiteux ou dans Gil Blas, Lesage sourit devant certaines réalités, se contentant le plus souvent de laisser apparaître dans l'action de ses personnages, plus que dans leurs discours, l'absurdité d'une croyance ou d'une certitude. Souligner l'ineptie par la mise en scène de l'ineptie, sans pourtant sombrer dans le grotesque : voilà ce à quoi s'emploie l'écriture lesagienne. On trouve partout dans les représentations offertes par Lesage des antithèses ou, du moins, des variations sur le propos de cette œuvre qui se plaît à jouer de ses contraires jusque dans la forme, dans le temps et dans les lieux qu'elle présente : elle tient de la relation de voyage et du récit historique autant que du roman picaresque et du roman de mœurs, et sa double narration s'inscrit dans des cadres sociohistoriques opposés (le Nouveau Monde des sauvages, des aventuriers, des flibustiers et l'Ancien Monde de l'aristocratie et de la bourgeoisie françaises).

Comme les détails ethnohistoriques, qui justifient l'action du protagoniste plus qu'ils ne font valoir leur vérité documentaire, les poncifs historiques que réunit Lesage dans son œuvre se vident eux-mêmes 
de leur contenu: n'en demeurent à la fin que le ton et le fil narratif qui les noue, composantes romanesques par lesquelles Lesage suggère toujours plus qu'il n'énonce. "L'ironie, cette impertinence nuancée, légèrement fielleuse, est l'art de savoir s'arrêter. Le moindre approfondissement l'anéantit ${ }^{23}$ ", écrit Cioran. Lesage aura su demeurer sur la ligne de partage.

EMMANUEL BOUCHARD

Université de Montréal 